\title{
THE ADAPTIVE WEB SERVER BASED ON ANT BEHAVIOR*
}

\author{
Ping Guo, Qin Xie \\ School of Computer Science, Chongqing University, Chongqing, 400044, P.R.China \\ E-MAIL: guoping@cqu.edu.cn
}

Abstract: This paper presents an approach to establish an adaptive Web site, which is inspired by the ant colonies foraging behavior. In this approach, artificial ants metaphorically guide users' activity and adaptively mark the most significant links, by means of the shortest route to target node. By the simulated experiment, we study those factors that influence Web user's behaviors, which can be useful for further theoretical research and algorithm realization.

Key words: ant colony algorithm, adaptive Web site, simulation

\section{INTRODUCTION}

Nowadays, World Wide Web has become the most dominant medium in these fields of information, communication and communion. The number of Web sites has increased rapidly, and the size of them has also enhanced. Consequently, it will be more difficult for the users to search from the large and complex information in the Web sites. Therefore, in the construction of Web sites, we should emphasize the importance of convenience and quickness.

One efficient solution is to establish adaptive Web sites through the study of user access pattern. The definition of an adaptive Web site is a site that automatically improves their organization and presentation based on user access pattern ${ }^{[1]}$. The adaptation can be realized in two ways. One is Dynamic Customization, which means it can adapt the site's presentation to

\footnotetext{
${ }^{*}$ This work was supported by National Natural Science Foundation of China, NO. 50378093.
} 
the needs of individual visitors, based on information about those individual, such as path prediction. The other is Optimization, which means it can improve the site's structure based on interactions with all visitors so that they can be visited more easily and conveniently, including Promotion and Demotion, Highlighting, Link, and Clustering ${ }^{[2]}$. Dynamic Customization focuses on individuals and is for a single user, while Optimization is for all users, and it focuses on the improvement of the site's structure and contents, in order that all the users can access them facilely and advantageously.

Proposed by M. Dorigo, ant colony algorithm ${ }^{[3]}$ imitates the action of ant colonies foraging behavior, which is a kind of bionic algorithm based on the natural environment, and is also for the adaptive problems. According to heuristic idea and through the inducement by pheromone, we can get the convergent and optimized solution. So far, ant colony algorithm has already been successfully applied in such fields as combinatorial optimization, computer network routing, continuous function optimization, robot path planning, data mining, and the optimization of electric network.

Based on the Web usage mining, this paper tries to apply the ant colony algorithm in the establishment of the adaptive Web site, and bring forward the Ant-based Adaptive Web site Algorithm (AAWA). Through the simulated experiment, we have studied the factors that influence the action of users, which include pheromone weight, pheromone evaporation coefficient, the number of Web users, and the amount of residual pheromone. The result can be significant to the further study and the realization of the algorithm.

\section{AAWA (ANT-BASED ADAPTIVE WEB SITE ALGORITHM)}

\subsection{The introduction of ant colony's behavior}

In nature, though the behavior of a single ant is extremely simple, when these ants act as a whole community, they are capable of solving complex problems in their daily life. Ethnologists have discovered that ants are capable of finding the shortest path from a food source to their nest without the use of visual information. And they are also able to adapt to the changes of the environment, for example, finding a new shortest path once the old one is no longer feasible due to a new obstacle. While walking from the food source to the nest, ants deposit on the ground a substance called pheromone, forming a pheromone trail. When a single ant moves at random and encounters those previously laid trails, it can feel the pheromone and is more 
likely to choose the thicker one and reinforces the trail with its own pheromone. The more the ants following a trail are, the more probability that this trail becomes attractive for the followers. The process is thus characterized by a positive feedback loop, where the probability that an ant chooses a path increases with the number of ants that chose the same path in the preceding steps.

In a sense, the process of surfing in Web is similar to that of the ant colonies foraging. In this paper, we consider users' visiting the Web as the ant colonies foraging process (the users are compared to the artificial ants, and the target node, the food source). Different from ants, visitors of Web do not have any communication. Each one obtains their own route to find their objective without the support of others that may have previously passed through the same path. So, in this article, we connect the users through imitating the ants' pheromone, which can make a single user find the target node more easily and quickly.

\subsection{Basic theory}

In the Web site, we can apply the ant colony theory to make Web users reach the target node through the shortest route (In this article, we name a Web site's page as a node and a link between pages as a route). The method is as follows: Within $t$, a Web user sets out from the homepage every $\Delta t$, and according to the staying time at each node and the pheromone density on each route, it selects the next node, and finally stops at the destination node. All users stop after $t$ and we can update the amount of pheromone at all nodes that the users who have reached the target have passed. Through several times of simulation (a process of $t / \Delta t$ users' finding target node within $t$ ), the users gradually concentrate on an optimized route. The detailed theory is the following:

We suppose there is a user visiting the Web site every $\Delta t$. The iteration $p$ is a number of the users visiting the Web site within $t$, namely, $p=t / \Delta t$. Here, $t$ is the duration of a simulation. The amount of pheromone $\tau_{\mathrm{ij}}(p)$ associated to a link $(i, j)$ is intended to represent the learned desirability of the choosing node $j$ when in node $i$. The initial amount of pheromone $\tau_{\mathrm{ij}}(0)$, at iteration $p=0$, is set to a small positive constant value or zero for all links. In our case the initial probability value which a user $(\mathrm{k})$ chooses from node $i$ to node $j \in N_{i}$ is obtained from the Web usage mining.

A probability value $p_{i j}^{d}$ expresses the goodness of choosing $j$ as next node when the target node is $d$, it has the constraint:

$$
\sum p_{i j}^{d}=1, j \in N_{i}, N_{i}=\{\text { neighbors }(i)\}
$$

We define the transition probability from the node $i$ to the node $j \in N_{i}$ : 


$$
p_{i j}^{d}(p)=\left[\tau_{,}^{\alpha}(p)\right]^{\alpha}\left[\eta_{j}(p)\right]^{\beta} / \sum\left\{\left[\tau_{,}^{d}(p)\right]^{\alpha}\left[\eta_{i}(p)\right]^{\beta}\right\} \quad j \in N_{i}, l \in N_{i}
$$

Where $\tau_{j}^{d}(p)$ is the amount of pheromone trail at the node $j$ at iteration $p$ for destination $d . N_{i}$ is the set of neighbors of the node $i$, and $\alpha$ and $\beta$ are both parameters that control the relative weight of residual pheromone trail and heuristic value. $\eta_{i}=1 / w t_{j}$ is the heuristic value of moving to node $j$ :

$$
w t_{j}=l t_{j}+v t_{j}
$$

Where $w t_{j}$ is the estimated time at the node $j$, including $l t_{j}$, the estimated time to get all information of the node $j$ to the browser at some velocity of the process; and $v t_{j}$, the estimated time to visit the node $j$.

In this paper, we define the distance between two neighboring nodes that users have passed as 1 . For example, if a user reaches the target node 2 through a route as $1 \mathrm{~A} \rightarrow 2 \mathrm{~A} \rightarrow 3 \mathrm{~A} \rightarrow 2 \mathrm{~B} \rightarrow 2$ (fig.1), then the distance between node $1 \mathrm{~A}$ and node $2 \mathrm{~A}$ is 1 and that between node $1 \mathrm{~A}$ and node 2 is 4 .

After a user has completed his visit, pheromone evaporation at all nodes is triggered, and then the user (k) deposits a quantity of pheromone $\Delta \tau_{i}^{d, k}(p)$ at each node that has been visited:

$$
\Delta \tau_{i}^{d, k}(p)=\left\{\begin{array}{cc}
1 /\left[\left(n l_{i}^{d, k}(p)+1\right) \sigma\right] & \text { if } i \in T^{d, k}(p) \\
\mathbf{0} & \text { if } i \notin T^{d, k}(p)
\end{array}\right.
$$

Where $T^{d, k}(p)$ is a set of all the visited nodes by user (k) to get to the destination node $d$, and $n l_{i}^{d, k}(p)$ is the distance between node $i$ and node $d$ in the $T^{d, k}(p) . \sigma$ is a parameter that can control and adjust the value of $\Delta \tau_{i}^{d, k}(p)$.

$$
\Delta \tau_{i}^{d}(p)=\sum \Delta \tau_{i}^{d, k}(p) \quad k=1, \cdots p, i \in T^{d, k}(p)
$$

The addition of the new pheromone by users and pheromone evaporation is implemented by the following rule applied to all nodes.

$$
\tau_{i}^{d}(p) \rightarrow(1-\rho) \tau_{i}^{d}(p)+\rho \Delta \tau_{i}^{d}(p) \quad \rho \in(0,1)
$$

Where $\rho$ is the pheromone trail decay coefficient.

\subsection{The algorithm}

At the beginning of the algorithm, all Web users are at the homepage of the Web site. There can be a user setting out from the homepage every $\Delta t$. When $t=0$, the first time of simulation begins, and when $t=T$, it ends. Therefore we can calculate the amount of pheromone at all the passed nodes that lead to the target node so as to update the pheromone of all nodes. Then the next similar simulation starts. If the times of simulation reaches the maximum $N C_{\max }$ set by us, or all the users choose the same path, the algorithm can be over. Ultimately, the optimized route that users have found 
is the optimized solution of the problem. The Steps are as follows:

(1) Applying the Web usage mining algorithm to get initial probability of the desirability of choosing the node $j$ when in the node $I\left(j \in N_{i}\right)$;

(2) Parameter initialization: Setting total time of a simulation as $T$, simulation times $N C=0$, the maximum of simulation times $N C_{\max }=\mathrm{N}$, $\tau_{\mathrm{ij}}(0)=\mathrm{C}, \Delta \tau_{\mathrm{ij}}=0$ and $t=0$.

(3) At the homepage of the Web site, a user (k) is generated every $\Delta t$ to find the destination node (in this paper, we limit ourselves to one destination node).

(4) Each user (k) at the node $i$ choose the next node randomly as gambling at a rotary table until they find the target node. (The rotary table gamble way is that we add those choice probabilities to get some accumulative probabilities, and then generate a random number which is in the domain of some accumulative probability, and the node corresponded the accumulative probability is the next node that will be selected.)

(5) When the user $(\mathrm{k})$ reaches the destination node, he stops and calculates, through the formula (4), the amount of pheromone $\Delta \tau_{i j}^{k}(p)$ that is deposited at each visited node.

(6) A time of simulation finishes when $t=T$. Resetting $N C=N C+1$ and $t=0$, through formula (5), we compute the total amount of pheromone at each visited node deposited by all the users that have reached the target node, and then update pheromone of all nodes according to formula (6).

(7) Recalculating the probability of choice from node $i$ to node $j\left(j \in N_{i}\right)$ by using formula (2), and replacing the existed probability.

(8) If $N C<N C_{\max }$ or no convergence phenomenon exists, then returning to step (3). Otherwise, inputting the shortest route and the algorithm is over.

\section{SIMULATED EXPERIMENT AND THE ANALYSIS OF THE RESULT}

In this experiment, we simulate the structure of the Web site as Fig.1. We set the duration of each simulation as $t=100 \mathrm{~s}$ and the interval between two users who are generated as $\Delta t=5 \mathrm{~s}$, so $\mathrm{p}=\mathrm{t} / \Delta \mathrm{t}=20$, meaning there are 20 users visiting the Web site in each simulation. The probability $\mathrm{P}(1 \mathrm{~A}, 2 \mathrm{~A})$ with which the users go along the route $1 \mathrm{~A} \rightarrow 2 \mathrm{~A}$ is fixed as $0.4, \mathrm{P}(1 \mathrm{~A}, 2 \mathrm{~B})$ as 0.4 , and $\mathrm{P}(1 \mathrm{~A}, 2 \mathrm{C})$ as 0.2 . Node 2 is the objective.

Through the simulated experiment, we select the representative data as follows:

(1) $\tau_{\mathrm{ij}}(0)=0, \mathrm{t}=100 \mathrm{~s}, \Delta \mathrm{t}=5 \mathrm{~s}, \alpha=2, \beta=3, \rho=0.7, \sigma=2$

(2) $\tau_{\mathrm{ij}}(0)=0, \mathrm{t}=100 \mathrm{~s}, \Delta \mathrm{t}=5 \mathrm{~s}, \alpha=1, \beta=2, \rho=0.7, \sigma=1$

(3) $\tau_{\mathrm{ij}}(0)=3, \mathrm{t}=100 \mathrm{~s}, \Delta \mathrm{t}=5 \mathrm{~s}, \alpha=1, \beta=2, \rho=0.7, \sigma=1$ 
Through the imitated experiment, we are able to observe that the third group of data is the best. From Fig. 2, we find the choices of the parameters have a great influence on the final performance of the algorithm. The parameters mainly include $\alpha, \beta, \rho, \sigma$ in this algorithm. $\alpha$ determines the relative importance of the remaining pheromone, $\beta$ represents the relative importance of the heuristic information, $\rho$ is the degree of pheromone evaporating, $\sigma$ is the parameter that controls and adjusts the value of released pheromone at each node. The higher $\alpha$ is, the higher the probability will be, with which Web users choose the route that has been passed before. That makes the algorithm converge more quickly, but meanwhile the search may be stagnated much earlier, and even leads to the local optimal solution. The higher $\beta$ is, the easier users will choose the node where the previous users have stayed shorter. When $\rho$ is lower, pheromone usually decays more slowly, and the influence of pheromone increment will be weakened, so the function of positive feedback is reduced; when $\rho$ is higher, some time later, pheromone on all routes is of the similar size. The accumulative knowledge cannot be utilized fully. When $\sigma$ is higher, the effect of pheromone increment is not obvious, and the function of positive feedback is reduced, too. Therefore, studying the disposition of the parameters $\alpha, \beta, \rho$ and $\sigma$ plays a very important role in solution of practical problems.

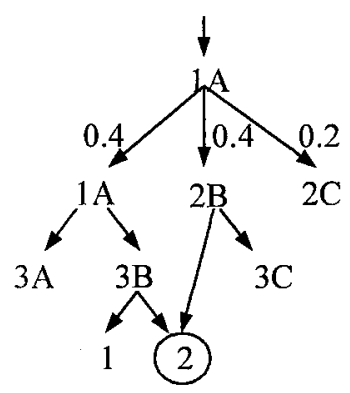

Figure 1.The structure of a Web site with two routes to the same target node

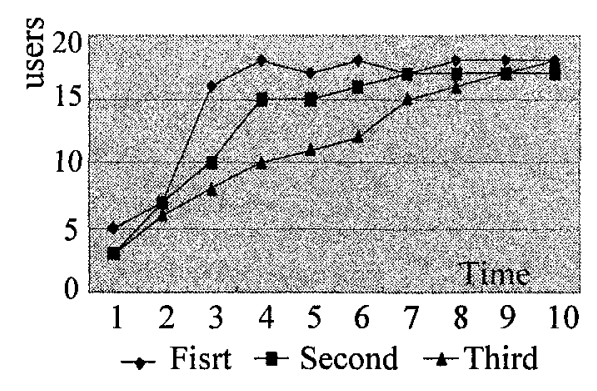

Figure 2. Evolution of the number of users reaching the target node through the shortest route

\section{ALGORITHM IMPROVEMENT}

In the above simulated experiments, from a large amount of experimental data we found that the algorithm converge when $N C \in[\mathbf{2 , 4}]$, which easily leads to local optimal solution. Therefore, we improve AAWA algorithm in three aspects of the following:

(1) Improving the ways of counting the amount of pheromone

When ant colony algorithm is applied in TSP (Travelling Salesman Problem), it uses average method to compute the amount of pheromone, 
namely, the total length that the ant has passed divided by the total amount of pheromone. So, in this paper, we improve the formula (5) that is used for counting the amount of pheromone at every node:

$$
\Delta \tau_{i}^{d, k}(p)= \begin{cases}1 /\left(\left(L_{k}(p)+1\right) \sigma\right) & \text { if } i \in T^{d, k}(p) \\ 0 & \text { if } i \notin T^{d, k}(p)\end{cases}
$$

Where $T^{d, k}(p)$ is a set of all the nodes passed by the user(k) until reaching the target node $d . L_{k}(p)$ is the total length passed by user(k) until reaching the target node $d$.

The amount of pheromone at these nodes that all the users has passed for the target node is the same through the above formula, unlike the formula (5), in which the nearer to the target node, the larger the amount of pheromone is. Meanwhile it makes some pheromone too centralized and neglects other routes to the target node, which would result in reducing search space or bogging in local optimized solution.

(2) Strengthening the effect of the users that have passed the optimized routes to the target nodes

After every simulation, if every user's pheromone overlapped on the route, the users, who find the worse route, may give their effects on the pheromone's distribution of the system. Under this situation, the advantage of the best route will not be shown. And so we should find the feedback from the users who use the shortest route in every simulation in order to avoid the useless information's interfere. We can change formula (6) into:

$$
\tau_{i}^{d}(p) \rightarrow(1-\rho) \tau_{i}^{d}(p)+\rho \Delta \tau_{i}^{d}(p)^{\text {Best }}
$$

(3) Limiting the pheromone density

For the existence of the positive feedback effect and pheromone's gradual evaporation, when the algorithm is bounded in the local optimal solution, the pheromone density on the route has the absolute advantage to other routes. In that case, it is hard for the algorithm to escape from the local optimized solution. Therefore, we must limit the maximum and minimum of the pheromone density on the route, such as $\left[\tau^{d}(p)_{\min }, \tau^{d}(p)_{\max }\right]$. If the amount of pheromone on any route is beyond the range, we should force the value to be the fixed maximum or minimum ${ }^{[4]}$.

Based on the above three aspects, we have improved the AAWA algorithm and made the simulated experiment. The applied data is as follows:

$$
\tau_{\mathrm{ij}}(0)=5, \mathrm{t}=120 \mathrm{~s}, \Delta \mathrm{t}=4 \mathrm{~s}, \alpha=1, \beta=2, \rho=0.7, \sigma=1
$$

The result is showed in Fig.3. We find that improved algorithm has an advantage in convergence, so the probability of being into the local optimal solution is relatively less.

Fig. 2 and Fig. 3 show that the number of users that reach the target node through the shortest route is an average value, not something individual. This is a result of the random feature: on the one hand, the algorithm itself is 
heuristic and random, and on the other hand, the staying time $w t_{j}$ at every node is also generated in a random way. Therefore, to find the average of the several numbers of users that reach the target node through the shortest route can reduce the influence of the random feature.

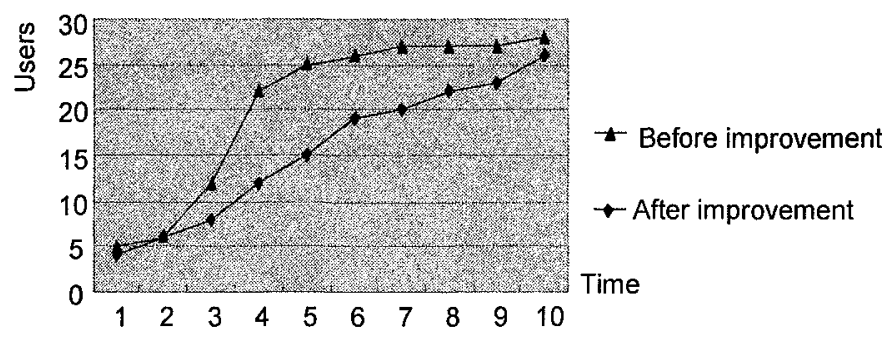

Fig. 3. Evolution of the number of users reaching the target node through the shortest route

\section{CONCLUSIONS}

In this paper, we combine the ant colony theory to adaptive Web techniques as a new method under the scientific research of Web Intelligence. In this way, the users can get to the target nodes through optimized routes. However, either ant colony theory or adaptive Web techniques need further development, and the new method still wants to be studied, in order that it can play more important role in the building of adaptive Web sites.

\section{REFERENCES}

[1] Perkowitz, M., Etzioni, O. Adaptive Web sites: an Al challenge. Proceedings of the IJCAI-97. Nagoya, Japan, 1997, 23-29.

[2] Perkowitz, M., Etzioni, O. Adaptive Web sites: automatically learning from user access patterns. In Proceedings of the Sixth Int. WWW conference, 1997.

[3] Dorigo, M., Garnbardella, L. Ant colony system: a cooperative learning approach to the traveling salesman problem. 53-66 IEEE Trans. Evolutionary Computation 1997, Vol.1(1), 53-66.

[4] Thomas, S., Holger, H., MAX-MIN Ant System. Future Generation computer Systems. 2000, Vol.16(8), 889-914.

[5] Wesley, M.,TELES,Li, W.. AntWeb- The adaptive Web server based on the ants' behavior. Proceedings of the IEEE/WIC International Conference on Web Intelligence (WI'03). 2003, 558-561. 